\title{
Avaliação Empírica da VMTools-RA: uma Arquitetura de Referência para Ferramentas de Variabilidade de Software*
}

\author{
Leandro Flores da Silva ${ }^{1}$ (mestrando), Edson OliveiraJr ${ }^{1}$ (orientador) \\ ${ }^{1}$ Mestrado Acadêmico em Ciência da Computação \\ Programa de Pós-Graduação em Ciência da Computação \\ Departamento de Informática - Universidade Estadual de Maringá (UEM) \\ Avenida Colombo, 5790 - CEP 87020-900 - Maringá - PR - Brasil \\ Ingresso: 03/2018 - Qualificação: 04/2019 - Previsão Defesa: 02/2020 \\ leandroflores7@gmail.com, edson@din.uem.br
}

Resumo. Arquitetura de Referência (AR) é um conceito que tem como objetivo concentrar as funcionalidades de um domínio de aplicação específico, fornecendo uma estrutura arquitetural genérica para o desenvolvimento de novos sistemas ou a evolução de sistemas existentes. Atualmente, diversas ARs vêm sendo propostas para diferentes contextos. Neste sentido, foi projetada a $V M T o o l s-R A$, uma AR para ferramentas de variabilidade de software. A VMTools-RA é composta de seis visões incluindo quatro pontos de vista arquiteturais. Tal AR foi avaliada de forma qualitativa e por meio de um experimento controlado. Este trabalho tem o objetivo de apresentar uma proposta de avaliação empírica da VMTools-RA, com base no projeto $e$ desenvolvimento de um ambiente (SMartyModeling) de gerenciamento de variabilidades para Linha de Produto de Software (LPS) modelados em UML, operados pela abordagem SMarty. Uma Revisão Sistemática da Literatura (RSL) vem sendo conduzida para compreender o estado da arte em relação à avaliações de AR. O SMartyModeling é composto de duas versões. A versão inicial foi desenvolvida e avaliada qualitativamente em 2017 e quantitativamente em 2018, sendo possível identificar algumas limitações. A segunda versão do SMartyModeling vem sendo desenvolvida, também construída a partir da instanciação da VMTools-RA. Portanto, este trabalho apresenta uma proposta para a avaliação da VMTools-RA sobre a perspectiva de uma arquitetura instanciada e implementada a partir dela. Neste sentido, a RSL é fundamental para compreender, analisar e projetar as avaliações, que serão realizadas sobre uma arquitetura instanciada a partir da VMTools-RA: a segunda versão do SMartyModeling.

Palavras-Chave: Avaliação Empírica. Arquitetura de Referência. VMToolsRA. SMartyModeling.

Eventos CBSoft: SBES e SBCARS

\footnotetext{
* O presente trabalho foi realizado com apoio da Coordenação de Aperfeiçoamento de Pessoal de Nível Superior - Brasil (CAPES) - Código de Financiamento 001.
} 


\section{Caracterização do Problema}

Com o objetivo de facilitar o desenvolvimento de arquiteturas para novos sistemas de um determinado domínio, a comunidade da Engenharia de Software (ES) propôs o conceito de Arquitetura de Referência (AR) [Cloutier et al., 2010]. Em geral, uma AR é considerada uma estrutura que, além de agregar comportamento, é a base a partir da qual as arquiteturas de software de sistemas de um domínio específico são construídas, empregando o conceito de reuso [Angelov et al., 2009].

ARs vêm sendo propostas para diversos contextos, como navegadores web [Grosskurth e Godfrey, 2005], Internet of Things (IoT) e ambientes educacionais [Fiorovanti et al., 2010]. O trabalho de Allian (2016) apresenta a VMTools-RA, uma AR para ferramentas de variabilidade de software. E por sua vez, a VMTools-RA serviu como base para a instanciação da arquitetura de um ambiente voltado para a modelagem de Linha de Produto de Software, o SMartyModeling [Silva, 2017].

A avaliação empírica de ARs é fundamental, pelo fato de contribuir com descobertas e evidências para construir uma base de conhecimento mais sólida, consistente e confiável. O trabalho de Nakagawa et al. (2014) apresenta o processo ProSA-RA, para a especificação de ARs, que define uma etapa de avaliação baseada em técnicas de inspeção dirigidas à detecção de defeitos nos documentos da AR, não especificando avaliações empíricas mais detalhadas.

A VMTools-RA foi avaliada inicialmente por meio de um estudo qualitativo, sendo utilizado um checklist denominado Framework for Evaluation of Reference Architectures (FERA). O estudo seguiu as recomendações descritas pela etapa de avaliação de ARs definida pelo ProSA-RA, sendo respondido por especialistas em AR e Gerenciamento de Variabilidades [Allian, 2016].

Assim, este trabalho tem como principal objetivo avaliar empiricamente a VMTools-RA. No entanto, a proposta consiste em avaliar a VMTools-RA a partir do ponto de vista de uma arquitetura instanciada. Para isso, apresenta inicialmente a definição de uma segunda versão do SMartyModeling, com a arquitetura também instanciada a partir da VMTools-RA e considerando os pontos a melhorar identificados nas avaliações realizadas sobre a primeira versão. Também é proposta uma Revisão Sistemática para compreender o estado da arte em relação às avaliações realizadas sobre ARs e assim projetar as avaliações empíricas, em especial, pretendendo avaliar a VMTools-RA sobre a perspectiva de arquitetura instanciada a partir dela, a segunda versão do SMartyModeling.

\section{Fundamentação Teórica}

\subsection{Arquitetura de Referência}

A indústria e a academia têm atualmente empregado o conceito de Arquitetura de Referência (AR) para o desenvolvimento de sistemas para variados domínios [Nakagawa et al., 2011]. No entanto, o termo AR vem sendo apresentado com múltiplos significados e aplicações em distintos níveis de detalhes e de abstração [DoD, 2010]. 
O trabalho de Nakagawa et al., 2011 apresenta uma compilação dos conceitos relacionados, sintetizando que o termo AR refere-se à uma arquitetura que compreende o conhecimento sobre como projetar arquiteturas concretas de sistemas voltados à um domínio de aplicação. Portanto, uma AR é considerada um padrão pré-definido projetado para um contexto de negócio específico.

A AR deve possuir conhecimento, experiência e informação adquirida, reusando a estrutura, elementos e artefatos contemplados em projetos anteriores [MartinezFernandez et al., 2013]. Assim, deve incluir as regras de negócios, estilos arquiteturais, melhores práticas de desenvolvimento e os elementos de software que suportam o desenvolvimento de sistemas para esse domínio [Nakagawa et al., 2014].

\subsection{A Arquitetura de Referência VMTools-RA}

A Variability Management Tools - Reference Architecture (VMTools-RA) é uma AR proposta no trabalho de [Allian, 2016], sendo voltada para o domínio de ferramentas de variabilidade de software. A VMTools-RA tem como objetivo apoiar a fase de projeto arquitetural no desenvolvimento e evolução de ferramentas de variabilidade de software.

Os requisitos arquiteturais da VMTools-RA foram identificados a partir de um conjunto de 29 ferramentas de variabilidade de software. O projeto arquitetural da VMTools-RA é constituído de seis visões da AR organizadas em quatro pontos de vista arquiteturais. A visão geral da VMTools-RA, apresentada na Figura 1, abrange quatro conjuntos de elementos [Allian, 2016]: (i) Elementos do Gerenciamento de Variabilidade: composto das definições do modelo, decisão, evolução e validação da variabilidade; (ii) Elementos de Análise de Domínio: composto do gerenciamento de ativos de domínio, incluindo uma camada de middleware, permitindo que possíveis tipos de ferramentas sejam integrados; (iii) Elementos de Suporte: composto do versionamento, importação, exportação e persistência, incluindo um repositório que fornece mecanismos para persistir as informações da aplicação; e (iv) Elementos do Gerenciamento Organizacional: composto de atividades como Planejamento e Gerenciamento; Comunicação e Compartilhamento; Guia; Análise de Trade-off ; Notificação e Feedback.

\subsection{O Ambiente SMartyModeling}

SMartyModeling é um ambiente projetado para a modelagem de LPSs, desenvolvido no trabalho de Silva (2017). A primeira versão foi definida em [Silva, 2017] e oferece suporte aos diagramas UML de casos de uso e de classes. A segunda versão, em desenvolvimento, e que faz parte deste trabalho, amplia o suporte para os diagramas de componentes, atividades e sequência, incluindo mais atividades identificadas pela VMTools-RA.

O ambiente tem como principal objetivo realizar a definição de variabilidades utilizando modelos de diagramas UML, com suporte à abordagem SMarty, mas adaptável a qualquer outra baseada no metamodelo da UML. A abordagem SMarty é uma abordagem anotativa baseada em esterótipos UML para o Gerenciamento de Variabilidades, sendo composta de um perfil UML denominado SMartyProfile. Além 
disso, o ambiente tem o propósito de gerenciar artefatos variáveis e apoiar as principais atividades relacionadas ao Gerenciamento de Variabilidades. Portanto, as arquiteturas das duas versões do SMartyModeling foram instanciadas a partir da VMTools-RA.

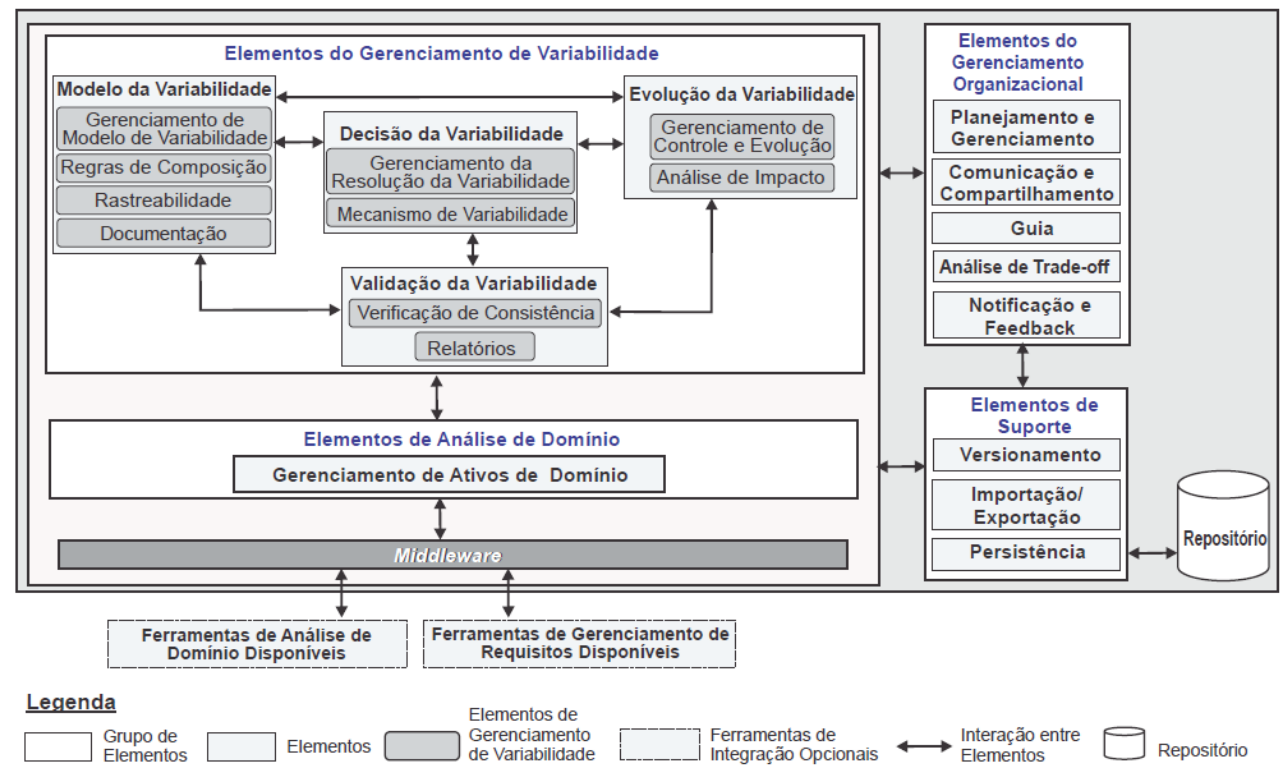

Figura 1. Visão Geral da VMTools-RA [Allian, 2016].

A primeira versão do SMartyModeling foi avaliada em duas oportunidades. A primeira, qualitativa, foi realizada por meio de um questionário construído conforme o modelo Technology Acceptance Model (TAM), sendo respondido por 10 especialistas. Como resultado, foi possível identificar os principais pontos a melhorar: limitação dos eventos para a criação de elementos, associações e a dificuldade para a modelagem de diagramas [Silva, 2017].

A segunda, realizada em 2018, teve como objetivo comparar quantitativamente o SMartyModeling e a ferramenta Astah. A avaliação foi realizada analisando a eficiência, definida como a razão entre o número de acertos e o intervalo de tempo, e a efetividade, definida com a razão entre o número de acertos e o número de elementos modelados. A avaliação foi realizada por 31 participantes que modelaram uma LPS nas duas ferramentas. Em síntese, os resultados obtidos forneceram indícios iniciais de maior eficiência do Astah, principalmente pelo fator tempo necessário para a modelagem e uma leve vantagem da efetividade do SMartyModeling, principalmente considerando o número de acertos.

Diante das limitações identificadas, uma segunda versão do SMartyModeling vem sendo desenvolvida, com o objetivo de contemplar mais elementos definidos pela VMTools-RA e, principalmente, melhorar a usabilidade conforme os pontos identificados. A segunda versão também tem a arquitetura instanciada a partir da VMTools-RA, incluindo a definição dos diagramas de componentes, sequência e atividades (suportados pela abordagem SMarty) e reestruturação das classes de modelagem e interface gráfica. 
O SMartyModeling é, dessa forma, imprescindível para a avaliação proposta neste trabalho, pelo fato de a avaliação empírica da VMTools-RA ser planejada a partir de uma instância e implementação de uma ferramenta de variabilidade de software.

\section{Trabalhos Relacionados}

O trabalho de Nakagawa et al. (2014) apresenta o ProSA-RA, que descreve uma etapa específica para avaliação de ARs. Tal etapa consiste em verificar a descrição arquitetural da AR com a intenção de detectar defeitos por meio de uma abordagem de inspeção baseada em checklists.

O trabalho de Zbick (2017) propõe uma AR para sistemas que apoiem o aprendizado em dispositivos móveis. Uma seção do trabalho apresenta uma avaliação baseada em cenários, realizada sobre três descrições de implementação construídas a partir da AR, sendo analisados os seguintes atributos de qualidade: portabilidade, adaptabilidade, reutilização e extensibilidade.

O trabalho de Avgeriou (2003) avalia uma AR a respeito dos atributos de qualidade, combinando duas técnicas: avaliação baseada em cenários e avaliação de protótipo arquitetural. O método aplicado foi realizado examinando o protótipo de arquitetura em operação e criando cenários para a avaliação de atributos de qualidade específicos de maneira qualitativa.

No entanto, o objetivo deste trabalho é avaliar uma AR sob a perspectiva de uma arquitetura instanciada e implementada a partir dela, diferente da proposta apresentada pelo ProSA-RA e nos trabalho de Zbick (2017) e Avgeriou (2003). Assim, a dificuldade em encontrar trabalhos que apresentem avaliações de AR motivaram uma Revisão Sistemática da Literatura (RSL) para compreender o estado da arte em relação às avaliações em AR. Em especial, com o objetivo de encontrar trabalhos que avaliem ARs a partir de arquiteturas instanciadas.

\section{Método de Pesquisa}

Como metodologia para o desenvolvimento deste trabalho foram definidas as seguintes etapas:

- Avaliar a primeira versão do SMartyModeling: o objetivo é realizar experimentos com a primeira versão do SMartyModeling, principalmente avaliando a modelagem de arquiteturas de LPS, fazendo uma comparação com as ferramentas normalmente utilizadas;

- Identificar as Limitações: conforme os resultados obtidos pelas avaliações sobre a primeira versão, é possível por meio de uma análise criteriosa, definir os principais pontos a melhorar para a segunda versão;

- Elaborar segunda versão do SMartyModeling: o objetivo é desenvolver a segunda versão do SMartyModeling. A segunda versão é projetada conforme os pontos identificados na etapa anterior. A arquitetura é construída a partir de uma instanciação da VMTools-RA. O processo detalhado, bem como as decisões e 
etapas no decorrer da instanciação, será descrito minuciosamente;

- Revisar Sistematicamente a Literatura (RSL): o objetivo é identificar o estado da arte em relação às avaliações sobre ARs. Foram selecionadas três bases eletrônicas indexadas, com a string de busca formada a partir dos termos Arquitetura de Referência e Avaliação; e

- Avaliar a VMTools-RA: as avaliações empíricas serão projetadas a partir de uma análise dos trabalhos retornados na RSL. As avaliações empíricas serão realizadas avaliando uma arquitetura instanciada a partir da VMTools-RA: a segunda versão do ambiente SMartyModeling;

\section{Estado Atual do Trabalho}

A Figura 2 apresenta uma linha do tempo com o desenvolvimento do trabalho. A linha do tempo inicia na apresentação da VMTools-RA, incluindo a definição das visões, pontos de vista arquiteturais e seus respectivos elementos, bem como um exemplo de instanciação de arquitetura [Allian, 2016]. A primeira versão do SMartyModeling foi apresentada como trabalho de conclusão de curso [Silva, 2017], sendo avaliada de maneira qualitativa sendo possível identificar os principais pontos a melhorar.

A partir de 2018, com o início do mestrado, foi realizado um experimento com o objetivo de comparar quantitativamente a primeira versão do SMartyModeling e a ferramenta Astah. O experimento controlado teve como objetivo comparar a eficiência e efetividade das duas ferramentas para modelagem de casos de uso. Simultaneamente, foi projetada a segunda versão do SMartyModeling, com a arquitetura também instanciada a partir da VMTools-RA e levando em consideração os pontos a melhorar identificados nas avaliações iniciais.



Figura 2. Linha do Tempo com o Desenvolvimento do Trabalho.

Portanto, a RSL está em execução, na fase de leitura dos títulos e resumos para a primeira seleção de estudos primários. Simultaneamente, o desenvolvimento da segunda versão do SMartyModeling vem sendo desenvolvida, em especial, a implementação das classes projetadas conforme os elementos definidos pela VMTools-RA. 


\section{Resultados Esperados}

O principal resultado esperado para este trabalho é a avaliação empírica da VMToolsRA. Como mencionado, a avaliação será projetada a partir de uma arquitetura instanciada a partir da VMTools-RA: a segunda versão do SMartyModeling. No entanto, existe a expectativa de outras contribuições, tais como: (i) construção da segunda versão do SMartyModeling, que proporcione suporte à modelagem de arquiteturas de LPS com base em UML, que não existe na literatura; (ii) descrição do processo completo para a instanciação dos elementos e das visões VMTools-RA para a arquitetura da segunda versão do SMartyModeling; e (iii) investigação e discussão sobre a viabilidade de avaliar uma AR a partir de uma arquitetura instanciada e implementada.

\section{Referências}

Allian, A. P. VMTools-RA: uma Arquitetura de Referência para ferramentas de variabilidade de software. Dissertação de Mestrado, Programa de Pós-Graduação em Ciência da Computação - Universidade Estadual de Maringá, 2016.

Angelov, S.; Grefen, P.; Greefhorst, D. A Classification of Software Reference Architectures: Analyzing Their Success and Effectiveness. 8th European Conference on Software Architecture (WICSA/ECSA), Cambridge, UK, p. 141-150, 2009.

Avgeriou, P. Describing, Instantiating and Evaluating a Reference Architecture: A Case Study. Default journal. University of Groningen, 2003.

Cloutier, R.; Muller, G.; Verma, D.; Nilchiani, R.; Hole, E.; Bone, M. The concept of Reference Architectures. System Engineer, v. 13, n. 1, p. 14-27, 2010.

Fioravanti, M. L.; Nakagawa, E. Y.; Barbosa, E. EducAR: Uma Arquitetura de Referência para Ambientes Educacionais. XXI Simpósio Brasileiro de Informática na Educação (SBIE) João Pessoa - PB - Brasil, 2010.

Grosskurth, A.; Godfrey, M. W. A Reference Architecture for Web Browsers. 21st IEEE International Conference on Software Maintenance (ICSM’05), p. 661-664, 2005.

Martinez-Fernandez, S.; Claudia Ayala, X. F.; Marques, H. M. REARM: A Reuse-Based Economic Model for Software Reference Architectures. Safe and Secure Software Reuse. 13th International Conference on Software Reuse (ICSR 2013), v. 54, p. 417-431, 2013.

Nakagawa, E. Y., Antonino, P. O.; Becker, M. Reference Architecture and Product Line Architecture: A Subtle but Critical Difference. 5th European Conference on Software Architecture (ECSA), p. 207-211, 2011.

Nakagawa, E. Y.; Guessi, M.; Maldonado, J. C.; Feitosa, D.; Oquendo, F. Consolidating a Process for the Design, Representation, and Evaluation of Reference Architectures. IEEE/IFIP Conference on Software Architecture - Washington, DC, USA: IEEE Computer Society, v. 1, p. 143-152, 2014.

Silva, L. F. SMartyModeling: um Ambiente de Modelagem para Linha de Produto de Software com base no Eclipse Modeling Framework. Monografia (Bacharelado em Informática), UEM (Universidade Estadual de Maringá), Maringá, Brasil, 2017.

Zbick, J. AWeb-based Reference Architecture for Mobile Learning: Its Quality Aspects and Evaluation. IEEE International Conference on Software Architecture Workshops, p. 230235, 2017. 